\title{
Vision Control System of Pipe Welding Robot
}

\author{
Lifang Tang \\ Department of Computer Engineering \\ Cangzhou Normal College \\ Cangzhou, Hebei Province, China \\ Tiandoudou2008@126.com
}

\author{
Chuanjin Wang \\ Department of Fine Arts \\ Cangzhou Normal College \\ Cangzhou, Hebei Province, China \\ 331757411@qq.com
}

\begin{abstract}
The author of this article designs a non-track automatic pipe welding robot, which mainly studies the image processing system of visual welding tracking. With the requirement of various interference noise and tracking accuracy in the welding process, this study adopts structure light CCD sensor checking system and image acquisition card processing images of computer software, in which sample filtering, edge checking, contour tracking, laser centerline's selection and checking of its characteristics. This processing method has the advantages of good effect and speedy processing that is able to meet the timely requirement of tracking system.
\end{abstract} CCD

Keywords- Pipe; Welding robot; Visual controlling; SCM;

\section{1. THEORY OF ROBOT VISION TECHNOLOGY}

The robot vision is with the rapid development of computer and electronic technology in the end of 1960s. In 1990 s, owing to the improvement of computer competence and the lowing price, with the rapid development of image processing hardware and $\mathrm{CCD}$ video camera, robot vision system, especially the optical sensing method and its rich amount of information has received extensive attention. It has many device types and principles of categories, taking welding field as an example. It has developed from the only welding position checking devices to the whole pattern recognition including groove, arc and welding pools. Pan Jiluan first proposed the mathematical model and its computer processing methods of the CCD image sensor to identify the welding surface horizontal temperature field, based on the calculation of classic welding thermal and the theory of blackbody radiation.

Through the temperature checking and contrastive experiments of real welding process, the theory analysis and mathematical are proved to be right. The calculated result after image processing is accurate, and the error analysis shows that the accuracy can be further improved. For this temperature identifying system structures simply and calculates speedy, it can be used in controlling the accurate, high-quality welding process. The more mature CCD tracking sensor used incorporates a structured laser illumination source and a vision light sensing video camera matrix. This non-contact optical front sensing technology has the characteristics both surface video camera structure light technology and line video arc light technology. With this method, the laser breaking welds we can get an ID image, then before the welding torch, we can quickly analyze the image to determine the position and geometric features of this weld. This technology is convenient and speedy, which do not require a lot of processing signals.

\section{SCM CONTROL SYSTEM}

The popular cycle welding robot in the course mainly requires controlling the body drive motor and the crossing drive motor, and some state functions are needed. Some parameters of the wire feeder and welder are coordinated by the controller. Vision signals are complicated, by complicated processing to get the related information of the welding groove. Therefore, the system controller is a multiinput and multi-output, in which the process is more complex and has to be achieved by a computer system. As the control center for the entire closed-loop system, the main achievements are the signal sensor processing, and output control to the completion of the closed-loop tracking. Taking into account the relatively harsh environment of industrial use and interference, the controller has strong anti-jamming capability. Furthermore, the main task of the timely controller by a microprocessor is a signal acquisition and processing, and outputs a control signal; the less information is exchanged directly with the human operator. According to the above features, the Internet 8098 micro controller is suitable for industrial control performance as the main chip of the control system. Compared with the general chips, this chip has the following characteristics:

- $\quad$ Ten-chip A / D conversion whose speed is $22 \mu \mathrm{S}$.

- Multi-channel high-speed input (HIS) and highspeed output (HSO) lines, in the CPU do not intervene in the case of real-time record of changes in the input signal and changes the output line level.

- 16-bit multiply and divide instructions in the speed of $6 \mu \mathrm{S}$.

- The chip clock is up to $12 \mathrm{MHz}$, speedy data processing. The whole SCM control system uses the STD model structure. The signal into the CPU or issued by the CPU needs to go through the buffer, and in real-time industrial control environment, needs the optical isolation. Input and output signals are switching signals and analog signals, so the input and output interface board includes: switching input boards, analog input board, switching output board and analog output board. 


\section{Vision RECOGNITION AND PROCESSING System}

In the process of tracking control using the vision sensing technology, the laser emits a point light source through the cylindrical lens into a line light source, projected onto the surface of the work piece, after reflection into the CCD, which will be on the work piece surface and the height of the two-dimensional information video signal into the image acquisition card, 8-bit digital signal after A / D conversion, and to interrupt the transmission into the computer memory via the PCI. Then it calls the image processing program for image preprocessing (filtering enhancement, etc.) and image post-processing (decimation weld centerline, characterized in detection), obtained after the deviation signal. The control signal is obtained by the control algorithm, the actuator control AC stepper motor for movement in all directions in order to achieve the purpose of real-time deviation adjustment [3]. The huge amount of information is processed by the image recognition and processing system, and the processing speed and accuracy has certain requirements and the scope of application of the system is quite extensive. Therefore, the present image processing system has a variety of structures, and more types of the commercialized products. By purpose, it can be divided into two major categories of special and general. The special system is designed for a special purpose, which generally requires a simple, rapid, accurate and economic system. So its structure is simpler than the general system, aim and function more accurate, and the scale smaller. Such as the Quantimet 720 image analyzer made by British, which is specifically for the quantitative analysis for the material of fibrous structure. The process of general image processing system can be showed in figure 1:

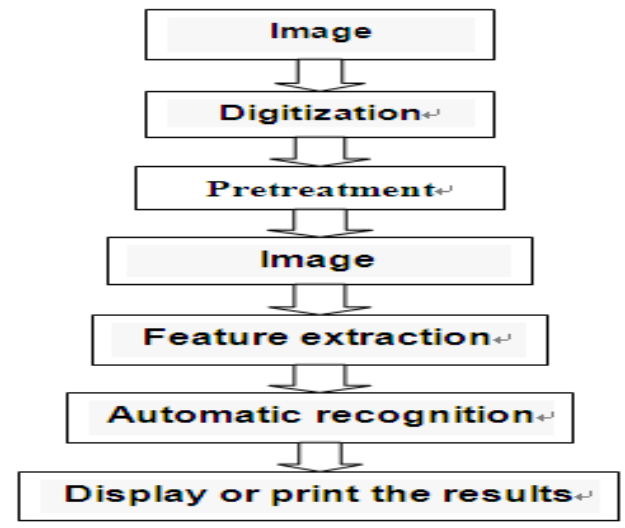

Figure 1. Working Process of Vision Treatment System

The image by the input device converted to a continuous analog electrical signal input system, first is converted into discrete digital signals by the A / D converter, in order to facilitate the operation of a digital computer processing. Owing to the various types of noise or distortion in the conversion from the actual scene to image information, the preprocessing is generally needed. The method can be used the above-described image enhancement or restoration technique, which can improve the image quality to some extent. Sometimes the image encoding compression technique is also used which can greatly reduce the amount of information, in order to achieve the reduction of the requirements of the computer storage capacity and the transmission channels.

If it is for identifying some information of the images automatically, detecting some peculiar patterns (target pattern) to identify the nature of the interesting objects in the image that needs to use the image segmentation technology, with which the image is divided into a set of simpler parts and extracts some information of the target features. They are organized with meaningful descriptions so that the computer can automatically identify or classify that is the basic content of pattern recognition. Finally, the computer processing results can be displayed or printed through the output device.

If the image processing is required to achieve the purpose of improving the picture quality, it is generally completed in pretreatment, which is usually referred as the image preprocessing system. This study is not only to improve the picture quality, but also requires the automatic identification, so we need the whole process of Figure 2, which is usually referred as the image processing and recognition system.

There are three basic parts to form the general image processing and recognition system:

- The image input device - the analog physical quantity (such as optical, ultrasound, X-ray and other information) of the image input is converted to electrical signal digitized for computer processing.

- Computer systems - to complete a variety of image processing and recognition software, is the core part of the system. Due to the large amount of information, Image processing must have external storages (such as disk, tape drives, etc.).

- Image output means - The intermediate results or the final results of the image processing are to be displayed or printed.

In the special image processing system, commonly some of the hardware of the image processor (features) carries out some specific processing, such as one-dimensional or twodimensional digital filtering, fast Fourier transform (FFT), the differential edge detection, statistics judgment classification. It often uses the structure of the parallel processing to improve the processing speed. Of course, the data and processes of the hardware of the processor are managed by the computer [5].

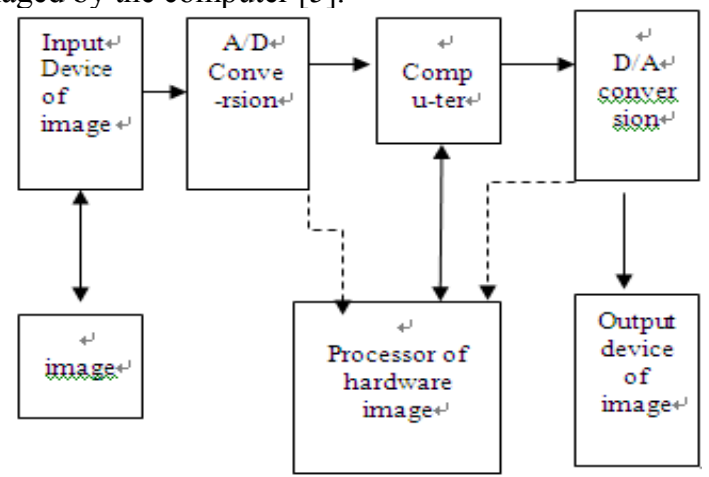

Figure 2. Basic Structure of System 


\section{TRACKING AND EXECUTING SYSTEM}

Tracking actuator as a unit of the closed-loop system, whose drive control is not too high, adopts the open-loop drive. The motor has two armature windings by converting the working winding to change the steering.

The distance of motor moving is $\mathrm{s}=\mathrm{v}$ ? $\mathrm{T}$, in which $\mathrm{s}$ can be controlled by the time adjustment method, i.e. FM can determine the velocity $\mathrm{v}$, and the regulator can control the energization time and running direction. Tracking the energization time of the motor is given by the control system using a pulse drive scheme in the process of time adjustment. Stepper motor speed is by the direct control of the frequency of the input pulse (printer port output pulse), the placement of the counter initial value Q, then the counter / timer the OUT0 generates a square wave frequency sequence, whose frequency is the $1.1931816 \mathrm{M}$ (clock frequency) / Q, stepper motor input pulse frequency $\mathrm{f}=1.1931816 \mathrm{M}$ (clock frequency) / 2Q. As the rotor teeth stepper motor revolution need to enter the number of pulses for MZK, its rotational speed is $\mathrm{n}=60 \times 1.1931816 \mathrm{M} /(2 \mathrm{Q} \times \mathrm{m} \times \mathrm{z} \times \mathrm{k})(\mathrm{rev} / \mathrm{min})$, wherein the number of phases $\mathrm{m}$ for the stator windings; $\mathrm{z} ; \mathrm{k}$ is a logic supply state the beat control mode $\mathrm{k}=1$, the six control mode $\mathrm{k}=2$. Under normal circumstances, the pulse frequency only in the less than (or equal to) operating frequency when the stepper motor, stepper motor in order to start, stop or reverse without the risk of losing steps. However, the stepping motor is very low in the frequency of starting and stopping the operation of the process, this frequency is typically $100 \mathrm{steps} / \mathrm{sec}$ to $250 \mathrm{steps} / \mathrm{sec}$, is much lower than the frequency of continuous operation [5]. In order to make the stepper motor without losing step phenomenon for fast operation, the variable speed control method is adopted..

\section{HM CONTROLLING SYSTEM}

In fact, all parts of the control drive of the entire visual sensor tracking system are integrated in a control box. Of course, as a complete welding tracking needing the welding power, constant speed wire feeder and the trolley, which are the basic equipments for gas shielded automatic welding machine.

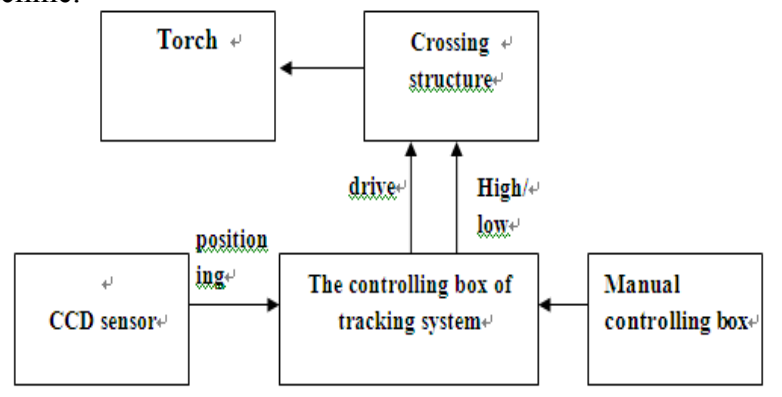

Figure 3. Actual System Constitution Diagram

\section{ACKNOWLEDGMENT}

The preferred spelling of the word "acknowledgment" in America is without an "e" after the "g". Avoid the stilted expression, "One of us (R.B.G.) thanks . .." Instead, try "R.B.G. thanks". Put applicable sponsor acknowledgments here; DO NOT place them on the first page of your paper or as a footnote.

\section{CONCLUSION}

Robot technology has gradually developed from the beginning of the simple open-loop industrial robots to the intelligent closed-loop control robot direction. The optical recognition technology in the development process has been more and more integrated into the robot technology that improved the intelligence of robots. Optical sensing method with rich amount of information has got extensive attention of scholars at home and abroad, such as the structured light method and the temperature field image recognition method, which are used better. Comparing the structured light method discussed herein is not only able to detect the center position of the welding, but also obtain the characteristic parameters of a cross-sectional shape and dimensions of the welding, and is applicable to the different shapes of the welding and the various welding methods. Of course, it has the disadvantages that they are easily influenced by spatter, smoking, steaming, arc and work piece rust, scratches, marks and oxide, etc., especially the V-shaped welding which has the interference of multiple reflections effect to structured light. All these factors will interfere with the CCD camera, affecting the quality of the image. How to eliminate the noise in order to obtain a high quality image is our further study to solve the problems.

\section{REFERENCES}

[1] Chen Qiang, Pan Jiluan. " The timely checking of CCD image sensor welding back temperature field ,". Journal of Qianhua University (Nature and Science),pp. 45-53, Feb 2010. (references)

[2] Charles Umeagukwu and James McCormick. "Investigation of an Array Technique for Robotic Seam Tracking of Weld Joint,".IEEE Transactions on Industrial Electronics Vol. 38 pp. 29-31,June 2007. (references)

[3] Song Guojun, Zhu Liumei, Wang Wei. " Research on vision welding tracking image ,’Welding Technology, pp.10-12,Jan 2003 (references)

[4] Ruan Qiuqi. "Data image processing,". Beijing: Press of El, ctronics and Industry, 2010. (references)

[5] Wu Yonghong, Kou Ziming. "The software achievement of data controlling functions of electricity machines ,"Shan Xi Machinery, pp. 14-15, Feb 2008 (references) 